\title{
La mise en œuvre de niveaux d'intervention pour la protection de la population, dans l'éventualité d'un accident nucléaire majeur. Passé, présent, avenir *
}

\author{
(Manuscrit reçu le 30 mai 1988)
}

\author{
B.W. EMMERSON **
}

\begin{abstract}
RÉSUMÉ
Depuis le milieu des années cinquante, l'énergie atomique a joué un rôle grandissant dans la réponse à la demande mondiale de production d'électricité. Durant cette période, se sont produits des incidents et des accidents, mais, dans la plupart des cas, leurs effets ont été limités au cadre de l'installation. Cependant, trois accidents ont été suffisamment sérieux pour comporter des conséquences pour la population. L'expérience que l'on a tirée de chacun d'entre eux a contribué de manière importante à l'élaboration de critères de réponse à une situation d'urgence, et de projets d'action concertée aux niveaux national et international. Dans cet article, nous résumons ces apports, dans la mesure où ils sont liés à la mise en œuvre de niveaux d'intervention pour la protection de la population, dans l'éventualité d'un rejet accidentel de substances radioactives dans l'environnement. Nous précisons les diverses mesures prises par les pays qui ont été touchés par les rejets de l'accident de Tchernobyl; nous passons en revue les actions entreprises par la suite par les organisations internationales compétentes afin de fournir des recommandations plus claires pour l'application des principes d'intervention et pour le développement des niveaux d'intervention dérivés, en particulier ceux visant à contrôler la consommation des denrées alimentaires contaminées, ou leur circulation dans le négoce international. Enfin, nous abordons les perspectives offertes concernant l'élaboration de principes d'intervention mieux harmonisés, fondés sur les recommandations élaborées au niveau international.
\end{abstract}

\section{ABSTRACT}

Since the mid-1950's nuclear energy has played an increasing role in meeting the world demand for electricity production. Although during this period incidents and accidents have occurred, in most cases their effect was confined to the plant. Three accidents, however, were sufficiently serious as to involve

* Communication présentée à l'International conference on Radiation protection in nuclear energy de I'AIEA, Sydney, 18-22 avril 1988 (IAEA-CN-51/85).

** Agence internationale de l'énergie atomique (AIEA), BP 100, A-1400 Vienne, Autriche.

Adresse actuelle: Health and safety executive, H.M. Nuclear installation inspectorate, Baynards House, 1 Chepstow Place, London, W2 4TF, UK. 


\begin{abstract}
off-site consequences for the public. The experience from each contributed significantly in the development of current emergency response criteria and planning arrangements at the national and international level. This paper summarizes these contributions as they relate to the development of intervention levels for the protection of the public in the event of an accidental release of radioactive materials to the environment. It indicates the various measures taken by those countries that were affected by the release from the Chernobyl accident and reviews the subsequent actions by relevant international organizations to provide more comprehensive guidance on applying the principles of intervention and developing derived levels, particularly those aimed at controlling the consumption of contaminated foodstuffs, or their movement in international trade. Finally, it considers the prospects for developing a more harmonized intervention approach based on the guidance now being completed at the international level.
\end{abstract}

\title{
CONSIDÉRATIONS GÉNÉRALES
}

1 - Depuis 35 ans, la contribution de l'énergie nucléaire vis-à-vis de la demande croissante d'électricité a été importante; la capacité nucléaire nette installée est de $298 \mathrm{GW}(\mathrm{e})$; l'expérience totale de fonctionnement est de 4600 années-réacteur [1]. Cependant, aucune entreprise humaine ne peut être sans risque; bien que l'industrie nucléaire ait conçu et appliqué des normes de sécurité que de nombreuses autres industries de haute technologie n'ont pas encore égalées, on doit reconnaître que, bien que la probabilité d'un défaut de fonctionnement conduisant à un accident soit faible, une telle possibilité ne peut être totalement écartée. Durant cette période, il s'est produit des incidents et des accidents, dans les centrales, dans le cycle du combustible nucléaire, ou dans d'autres activités; cependant, à quelques exceptions près, leurs effets sont restés localisés à l'intérieur de l'installation; un petit nombre seulement ont nécessité des mesures portant sur la zone extérieure au site. Trois, à Windscale (qui n'est pas une centrale électro-nucléaire), à Three Mile Island (TMI) et à Tchernobyl ont eu un impact important, au niveau national et international, sur l'élaboration des plans d'urgence, y compris les critères d'intervention, pour la protection de la population.

2-Par définition, dans un acccident, la source d'exposition échappe au contrôle; le système de limitation des doses, recommandé par la Commission internationale de protection radiologique (CIPR) [13], et inclus dans les normes fondamentales de protection de l'AIEA [2], ne s'applique donc pas. L'extension de l'exposition qui en résulte ne peut être limitée qu'au moyen d'une intervention adaptée. Les principes qui sous-tendent le système de limitation des doses peuvent, cependant, constituer la base de l'organisation de cette intervention. En particulier, deux des trois composantes de ce système, à savoir la justification et l'optimisation, jouent un rôle important dans l'aide aux décisions d'intervention; la troisième composante, les limites de doses, n'est pas applicable. Les niveaux d'intervention ont des buts tout à fait différents de ceux des limites de doses. Les limites de dose recommandées par la CIPR sont conçues pour s'appliquer, dans des conditions normales, à la somme des doses dues à une combinaison donnée de sources. Cette combinaison n'inclut pas - parmi d'autres - 
les expositions dues aux radionucléides présents dans l'environnement, à la suite d'accident. Les niveaux d'intervention sont liés de manière spécifique à la dynamique de l'action, ou aux mesures de protection envisagées; la nature et la chronologie de leur mise en œuvre dépendront des circonstances qui prévalent alors, y compris l'étendue du risque potentiel (tableau I).

\section{TABLEAU I}

\section{Voies d'exposition, phases de l'accident et mesures de protection pour lesquels on peut établir des niveaux d'intervention.}

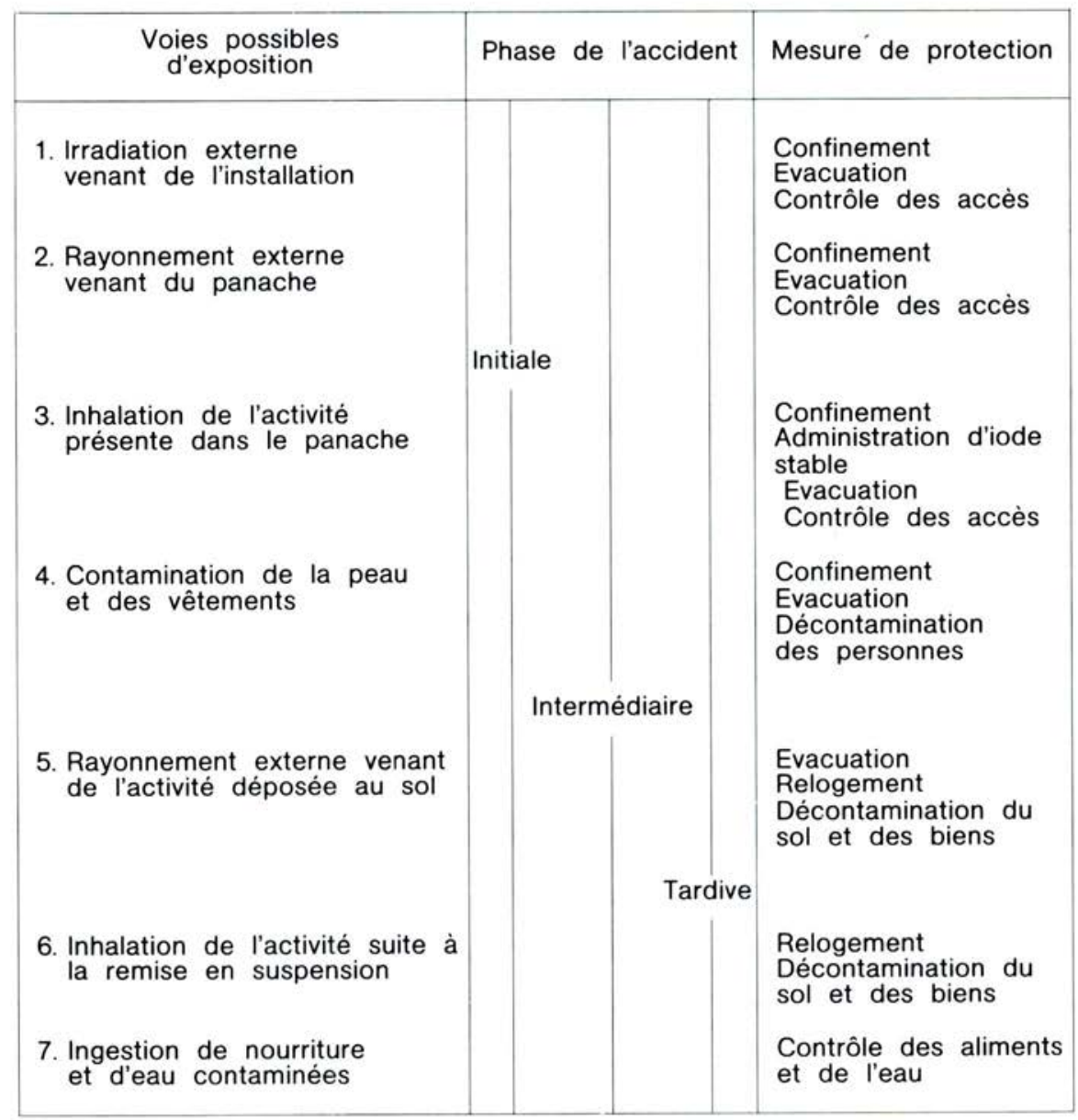

Note - L'emploi, pour les animaux, de nourriture stockée afin de limiter l'absorption de radionucléides par les animaux domestiques dans la chaîne alimentaire est valable pour n'importe laquelle des phases. 
On peut alors définir quantitativement les niveaux de risque prévus, sous forme de doses de rayonnement: selon les pays, ce sont les "niveaux d'intervention" (ILS), les "niveaux de référence d'urgence" (ERLS), ou les guides d'action de protection ${ }^{1}$ (PAGs). Les établir est une condition préalable importante pour la prévision d'une situation d'urgence: c'est sur ces niveaux que devrait se fonder toute décision d'exécution des mesures de protection durant un accident. On spécifie habituellement ces niveaux en équivalent de dose, car cette grandeur est considérée comme la grandeur dosimétrique appropriée pour exprimer le risque stochastique pour les individus. En cas d'incorporation d'une substance radioactive, on utilisera plutôt l'équivalent de dose engagé, ou l'équivalent de dose efficace engagé. On peut aussi exprimer ces niveaux sous forme d'activités et de concentrations dans l'environnement ( $p$ ar ex.: Bq m${ }^{-3}, \mathrm{~Bq} \mathrm{~m}^{-2}, \mathrm{~Bq} \mathrm{~kg}^{-1}$ ou $\mathrm{Gy} \mathrm{h}^{-1}$ ), (tableau II). Ces niveaux d'intervention dérivés constituent l'expression pratique des niveaux d'intervention; on peut les déterminer pour la gamme des radionucléides potentiellement importants qui peuvent être rejetés dans l'environnement, lors d'un accident nucléaire (fig. 1).

\section{TABLEAU ॥}

Grandeurs utiles pour les niveaux d'intervention dérivés.

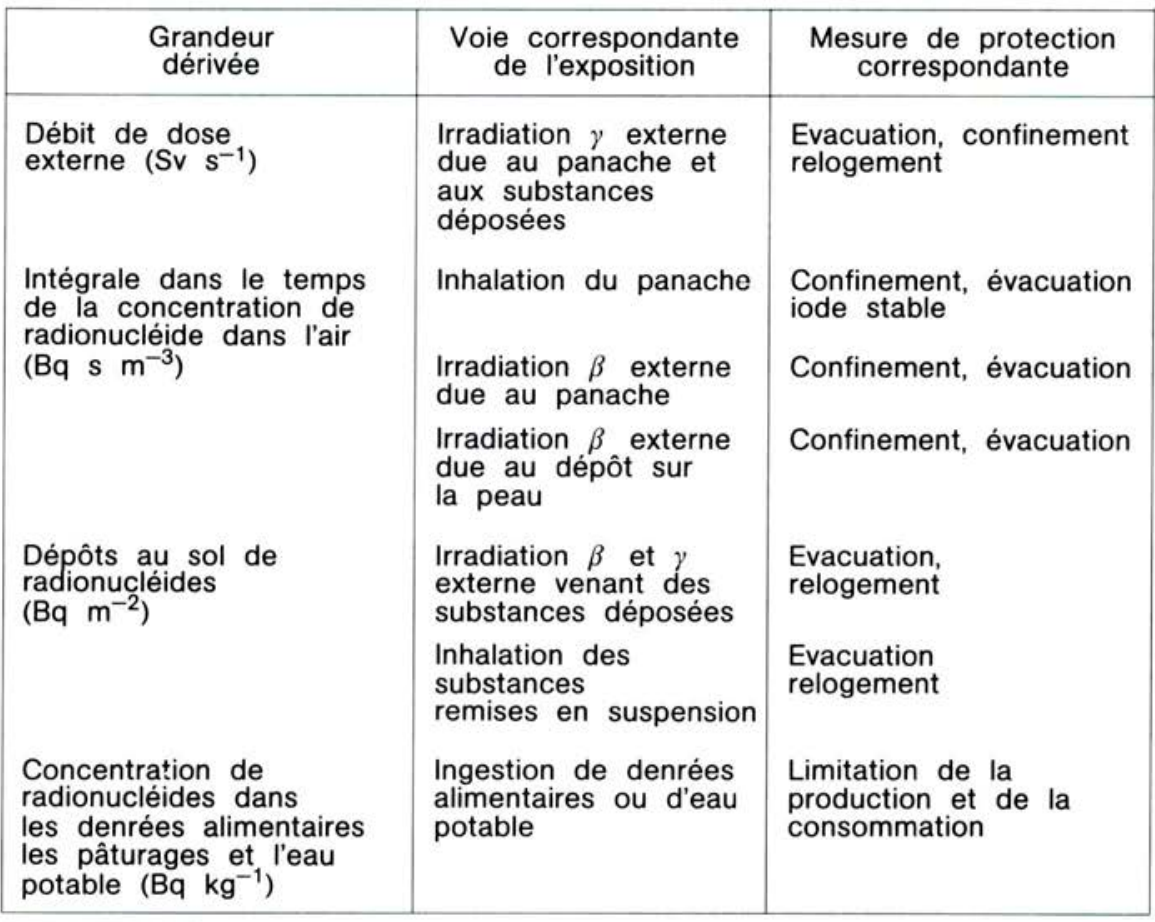

1. En anglais: ILs: intervention levels (CIPR, AIEA, OMS); ERLs: emergency reference levels (UK); PAGs: protection action guides ((USA). 
NIVEAUX D'INTERVENTION POUR LA PROTECTION DE LA POPULATION

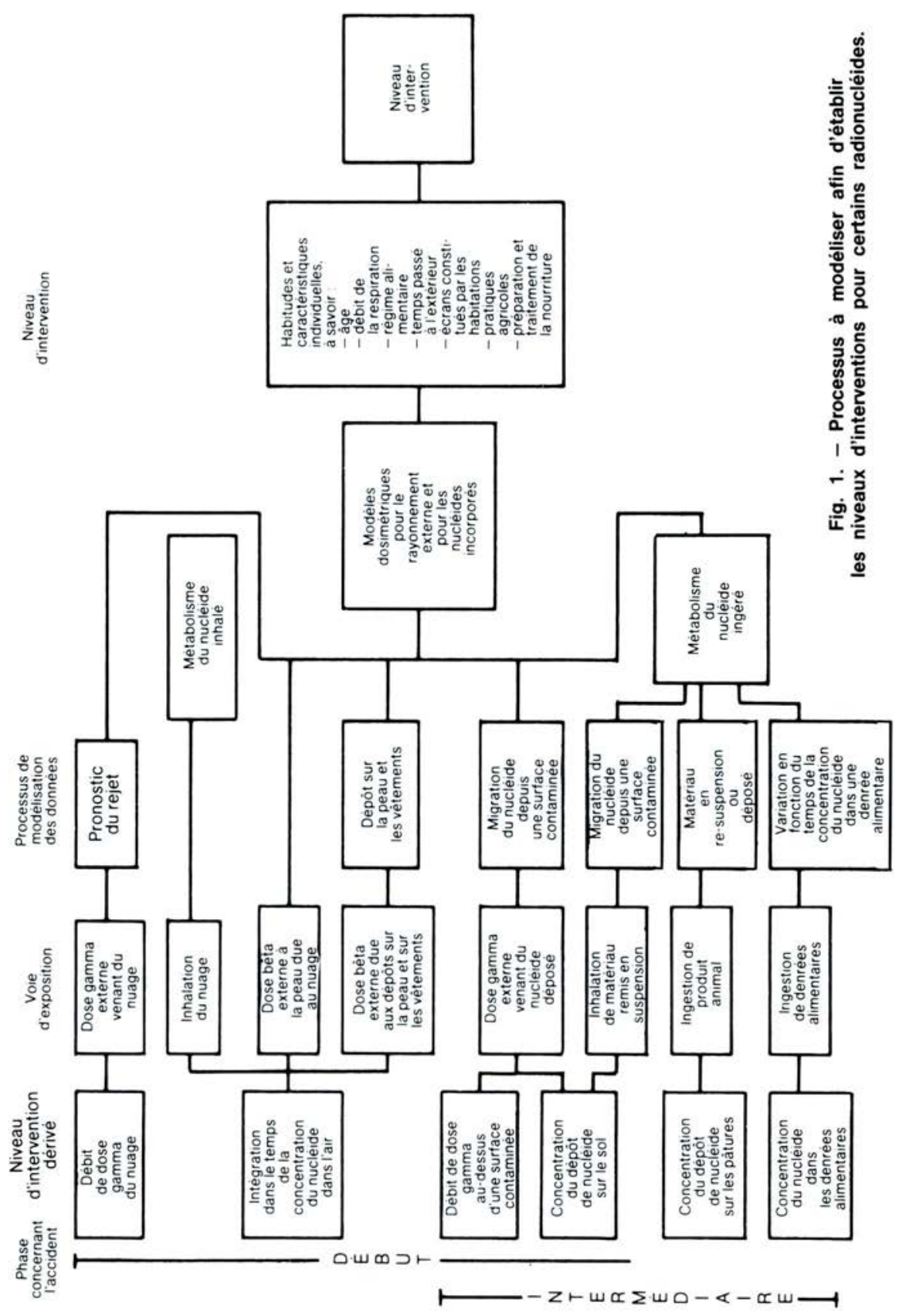

VOL. $24-N^{\circ} 1$ 


\section{L'“APRĖS-WINDSCALE”}

3 - Les valeurs de référence reliant les résultats du contrôle de l'irradiation à une dose potentielle étaient utilisées antérieurement au développement de l'industrie électro-nucléaire, mais elles concernaient avant tout les situations de défense militaire ou civile, plutôt que la protection normale du public, en particulier en ce qui concerne la protection contre la contamination de la chaîne alimentaire. L'incendie du réacteur $\mathrm{n}^{\circ} 1$ de production de plutonium à Windscale en 1957 [16], la libération de $20000 \mathrm{Ci}$ (740 TBq) d'iode 131, en même temps que d'autres produits de fission, ont mis, pour la première fois, en pleine lumière, la nécessité d'établir des critères pré-déterminés, constituant la base de l'élaboration des mesures de protection du public, dans l'éventualité d'une libération accidentelle de matière radioactive dans l'environnement. A la suite de l'accident de Windscale, le Medical research council de Grande-Bretagne a établi et publié une recommandation de niveaux de référence pour l'organisme entier et pour des organes particuliers; il a publié également un guide pour la détermination des niveaux dérivés dans l'air, le lait, le fourrage, pour toute une série de produits de fission [17]. Plus tard, le concept de niveau d'intervention a été adopté et étendu à divers niveaux nationaux et par les organisations internationales concernées. La collection sécurité de l'AIEA $n^{\circ} 55$ [3] résume les niveaux d'intervention et les niveaux dérivés applicables dans divers pays, au moment de l'accident de TMI.

\section{L'“APRĖS-THREE MILE ISLAND”}

4 - Contrairement à ce qui avait eu lieu lors de l'accident de Windscale, la fusion de l'unité 2 de la centrale électro-nucléaire de Three Mile Island, en 1979 , n'a pas conduit à une contamination sensible de l'environnement à l'extérieur du site [20]. Le résultat de cet accident a été, cependant, une plus grande prise de conscience de la possibilité de rejets importants à l'extérieur du site; durant les années suivantes, il a suscité la révision des guides donnant les principes de l'intervention pour la protection du public, et de son développement ultérieur aux niveaux national et international. Le concept initial d'une valeur unique de dose à laquelle il faudrait mettre en œuvre une mesure particulière de protection commença à ouvrir la voie à un concept plus réaliste et plus souple - celui d'une gamme de doses à l'intérieur de laquelle l'intervention doit être envisagée. Cela a conduit au concept, admis maintenant de manière générale, qu'il y a un niveau inférieur de dose au-dessus duquel il faut considérer l'introduction possible de mesures de protection appropriées; au-dessous de ce niveau, on considère généralement que les risques associés à leur introduction dépassent ceux qui sont associés à la dose; il y a de même un niveau supérieur de dose au-dessus duquel on prendra presque certainement les mesures prévues. Quand la dose prédite se situe entre ces deux niveaux, toute décision de mettre ou ne pas mettre en œuvre une mesure de protection particulière doit peser les risques potentiels et les coûts sociaux ainsi entraînés, et les comparer au risque radiologique.

5-A la fin de 1985, plusieurs organisations ont publié des guides concernant la conception et la préparation des plans d'intervention, et 
avaient donné le principe général d'élaboration des niveaux d'intervention. II s'agit de la CCE (1982) [9], de la CIPR (1984) [14], de l'OMS (1984) [22], de l'AIEA (1985) [4]. La CIPR a formulẹ ainsi les principes de base des plans d'intervention en cas d'accident:

- II faut éviter l'apparition d'effets non-stochastiques graves par la mise en œuvre de mesures de protection en limitant les doses individuelles à des niveaux inférieurs aux seuils d'apparition de ces effets;

- Il faut limiter le risque d'effets stochastiques, en introduisant des contre-mesures qui apportent un bénéfice positif net aux individus concernés;

- II faut limiter l'incidence globale d'effets stochastiques, autant que cela est raisonnablement possible, en diminuant l'équivalent de dose collectif.

Les recommandations comportent également les valeurs numériques des niveaux supérieur et inférieur de dose qui forment la base des mesures clefs de protection (confinement, mesures prophylactiques, évacuation), applicables lors des phases initiale et intermédiaire d'un accident (tableau III).

TABLEAU III

Niveaux d'intervention primaires (mSv).

Recommandations des organisations internationales compétentes, avril 1988, d'après $[4,9,14,22]$.

\begin{tabular}{|c|c|c|c|c|c|}
\hline \multirow{2}{*}{$\begin{array}{c}\text { Type } \\
\text { d'intervention }\end{array}$} & \multirow{2}{*}{$\begin{array}{c}\text { Organisation } \\
\text { consultée }\end{array}$} & \multicolumn{2}{|c|}{$\begin{array}{c}\text { Equivalent de dose } \\
\text { efficace à } \\
\text { l'organisme entier }\end{array}$} & \multicolumn{2}{|c|}{$\begin{array}{l}\text { Organe unique irradié } \\
\text { de manière } \\
\text { préférentielle }\end{array}$} \\
\hline & & Niveau bas & Niveau haut & Niveau bas & Niveau haut \\
\hline Confinement & $\begin{array}{l}\text { CIPR } \\
\text { AIEA } \\
\text { OMS } \\
\text { CCE }\end{array}$ & $\begin{array}{l}5 \\
5 \\
5 \\
5\end{array}$ & $\begin{array}{l}50 \\
50 \\
50 \\
25\end{array}$ & $\begin{array}{l}50 \\
50 \\
50\end{array}$ & $\begin{array}{l}500 \\
500 \\
250\end{array}$ \\
\hline $\begin{array}{l}\text { Administration } \\
\text { d'iode stable }\end{array}$ & $\begin{array}{l}\text { CIPR } \\
\text { AIEA } \\
\text { OMS } \\
\text { CCE }\end{array}$ & & & $\begin{array}{r}50 \\
50 \\
5 \\
50\end{array}$ & $\begin{array}{r}500 \\
500 \\
50 \\
250\end{array}$ \\
\hline Evacuation & $\begin{array}{l}\text { CIPR } \\
\text { AIEA } \\
\text { OMS } \\
\text { CCE }\end{array}$ & $\begin{array}{r}50 \\
50 \\
100 \\
100\end{array}$ & $\begin{array}{c}500 \\
500 \\
1000 \\
500 \\
(1000-\text { peau })\end{array}$ & $\begin{array}{l}500 \\
500 \\
- \\
300\end{array}$ & $\begin{array}{c}5000 \\
5000 \\
- \\
1500 \\
(3000 \text {-peau) }\end{array}$ \\
\hline Relogement & $\begin{array}{l}\text { CIPR } \\
\text { AIEA } \\
\text { OMS } \\
\text { CCE }\end{array}$ & $\begin{array}{l}50 \\
50 \\
>\mathrm{B} / \mathrm{G}^{\star} \\
-\end{array}$ & $\begin{array}{r}500 \\
500 \\
50 \\
-\end{array}$ & $\begin{array}{l}\text { Non } \\
\text { Non } \\
-\end{array}$ & $\begin{array}{l}\text { prévu } \\
\text { prévu } \\
- \\
-\end{array}$ \\
\hline $\begin{array}{l}\text { Contrôle des } \\
\text { aliments }\end{array}$ & $\begin{array}{l}\text { CIPR } \\
\text { AIEA } \\
\text { OMS } \\
\text { CCE }\end{array}$ & $\begin{array}{l}5 \\
5 \\
- \\
-\end{array}$ & $\begin{array}{l}50 \\
50 \\
- \\
-\end{array}$ & $\begin{array}{l}50 \\
50 \\
- \\
-\end{array}$ & $\begin{array}{c}500 \\
500 \\
- \\
-\end{array}$ \\
\hline
\end{tabular}

* $B / G$ : niveau dû à la radioactivité naturelle. 
II n'existe pas de valeurs de niveaux d'intervention dérivés reconnues au niveau international; cependant, les guides indiquent les mesures dans l'environnement ou les substances qui nécessitent leur emploi, ainsi que les unités à utiliser. Du point de vue collectif, ces guides représentent un concensus international général sur les principes d'établissement des niveaux d'intervention concernant la protection de la population, dans l'éventualité d'un accident nucléaire. Cependant, l'établissement de niveaux d'intervention pour des circonstances particulières demeure du domaine de la responsabilité des autorités nationales compétentes. Au moment de l'accident de Tchernobyl, un certain nombre de pays avaient établi des niveaux d'intervention à utiliser, dans le cadre des plans d'intervention d'urgence concernant leurs principales installations nucléaires, comme les centrales électronucléaires. Plusieurs d'entre eux, dont I'URSS (tableau IV) avaient mis en œuvre et publié des niveaux d'intervention portant sur toute une série de radionucléides [18 - 19, 25].

TABLEAU IV

Critère, en URSS, de décision sur la mise en œuvre de mesures protection de la population, dans l'éventualité d'un accident de réacteur, d'après [13].

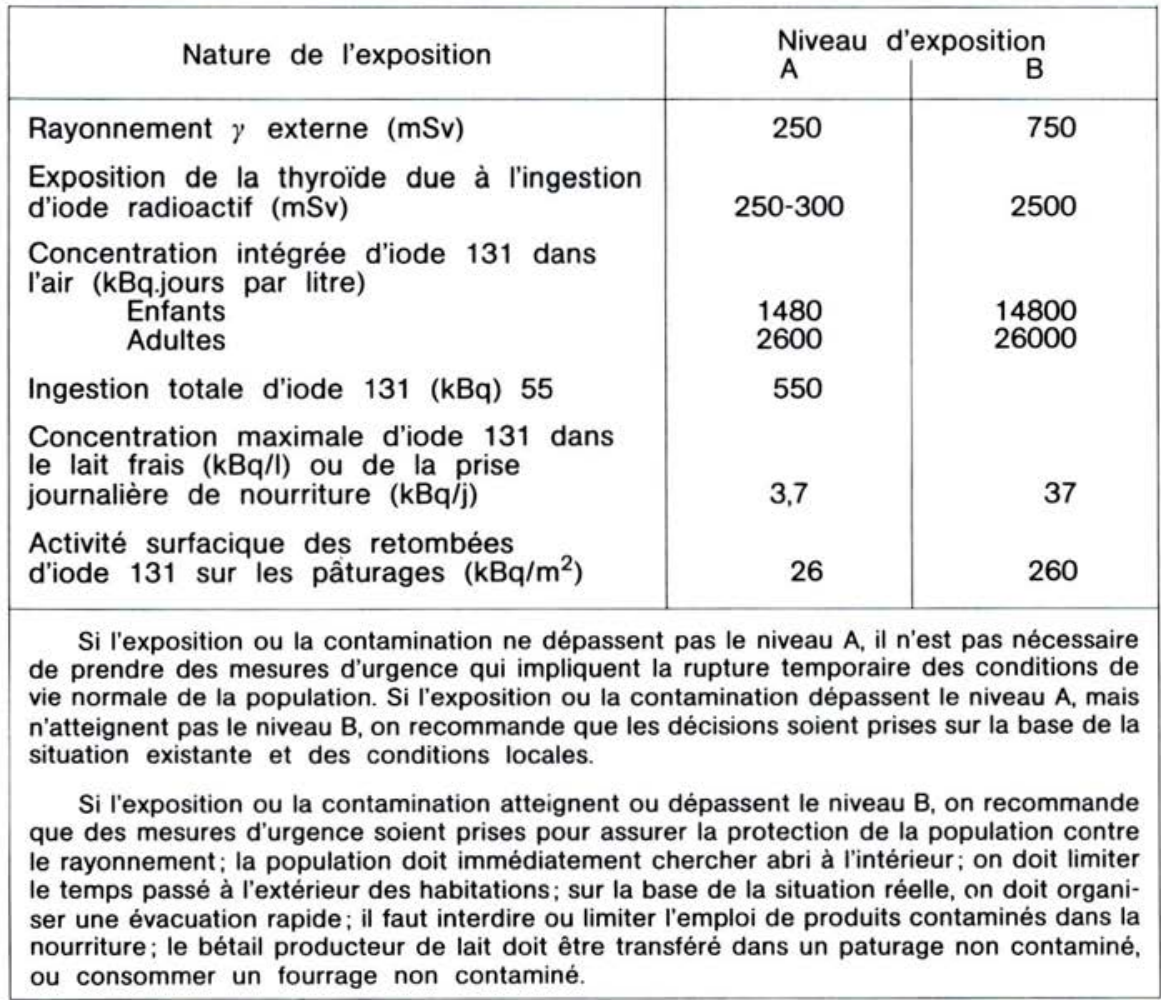




\section{L'“APRĖS-TCHERNOBYL”}

6-L'accident de la centrale électro-nucléaire de Tchernobyl le 26 avril 1986 a eu un impact majeur sur la manière dont on appréhende les plans d'intervention en cas d'urgence, aux niveaux national et international, l'impact dû - entre autres - au transport de substances radioactives à de grandes distances dans l'hémisphère nord, et au dépôt inhomogène qui en est résulté sur des étendues extrêmement vastes [5]. II existe un certain nombre d'accords bilatéraux ou multilatéraux [15] sur les rejets accidentels potentiels au-délà des frontières, lorsque des centrales électro-nucléaires sont situées dans la zone frontière entre pays voisins (par exemple SuèdeDanemark, Tchécoslovaquie - Autriche) ou dans une région où existent des intérêts économiques communs (par exemple, Conseil d'assistance économique mutuelle (CAEM), Commission des communautés européennes (CCE) ; par contre, aucune instruction internationale, aucun plan national d'urgence ne prend en compte le type d'accident qui pourrait disperser des quantités aisément mesurables de radionucléides sur d'importantes régions de l'Europe, avec des quantités détectables sur la plus grande partie de l'hémisphère nord. Les dispositions prises au niveau national, et les instructions publiées par les organisations internationales, sont, avant tout, conçues pour des rejets relativement bien définis, provenant de sources situées à des endroits spécifiques à l'intérieur des frontières nationales; elles ne concernent pas les conséquences, au-delà des frontières, de contaminations ayant leur origine à l'intérieur du pays.

7 - Les pays directement affectés par les retombées ont dû effectuer durant le stade initial d'évaluation - un effort considérable d'identification des radionucléides et de définition des zones où le niveau de contamination était suffisamment élevé pour justifier une action de protection. Les réactions des autorités nationales ont été très variables: d'un simple renforcement des programmes normaux de surveillance de l'environnement, sans introduction de mesures de protection, jusqu'à l'interdiction de denrées alimentaires précises; dans un cas, les autorités ont fourni de l'iode stable, à titre de précaution contre un apport excessif de radioiode à la thyroïde [24]. Cette approche différente des divers pays, particulièrement en ce qui concerne les niveaux pour lesquels des mesures de protection ont été mises en œuvre, ne peut s'expliquer ni par la variation des niveaux de contamination ou de leurs éventuelles conséquences radiologiques, ni par des différences locales de mode de vie ou d'alimentation. II est certain que des facteurs supplémentaires ont pesé sur la décision: le plus important a été de nature politique et économique (proximité d'élections, compensation portant sur des produits agricoles, etc...), plutôt que la protection contre les rayonnements. En outre, il y a eu une interprétation erronée des guides de protection radiologique existants: par exemple, on a confondu les raisons fondamentales sur lesquelles reposent les niveaux d'intervention avec celles qui sont liées aux limites de dose. La figure 2 illustre la gamme des niveaux dérivés d'intervention adoptés par quelque 31 pays pour le césium 134 et le césium 137 dans les denrées alimentaires. Les réactions très diverses des autorités nationales, associées à un manque d'information logique et claire du public (particulièrement en ce qui concerne la contamination des 
aliments et de l'environnement, les doses de rayonnement et leurs effets) ont, sans nul doute, surajouté, dans certains pays, une confusion et une anxiété inutiles [6].

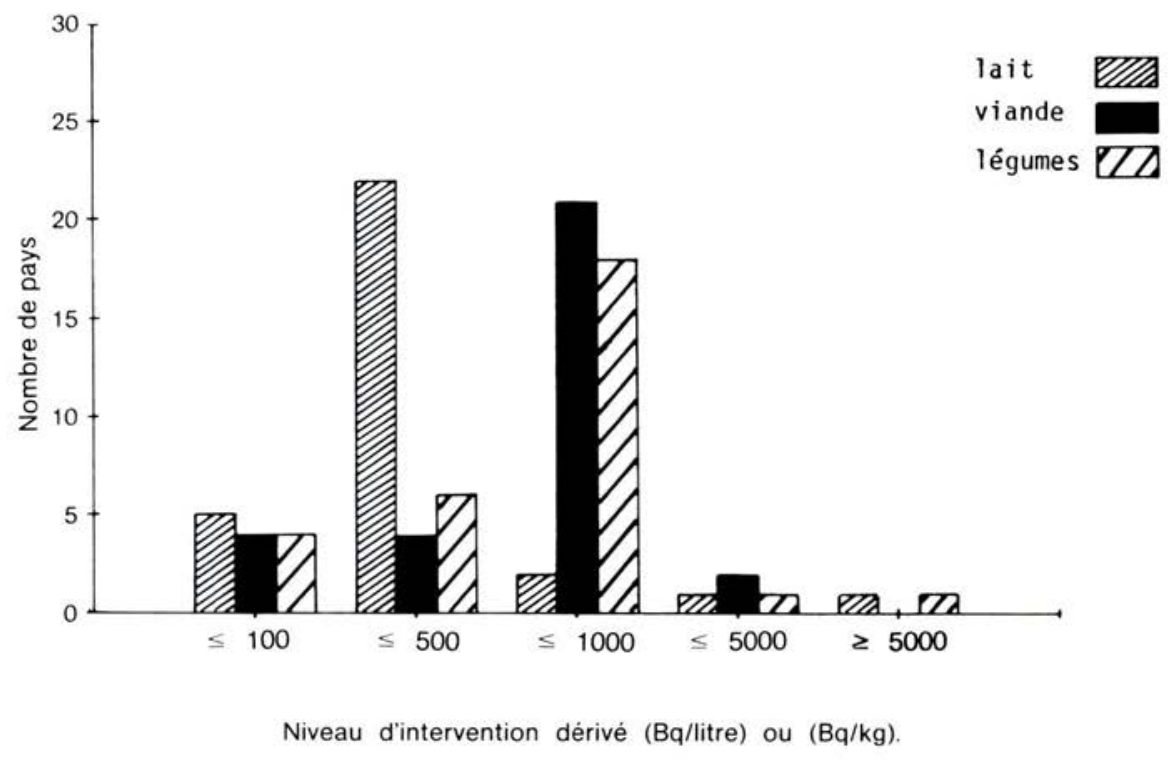

Fig. 2. - Variation des niveaux d'intervention dérivés. Analyse des valeurs portant sur 31 pays au 31 mai $1986\left({ }^{134} \mathrm{Cs}\right.$ et $\left.{ }^{137} \mathrm{Cs}\right)$.

8-Le problème immédiat de l'information concernant la protection contre les radionucléides entrant dans la chaîne alimentaire a démontré l'urgence d'établir au niveau international un guide clair consacré aux principes, aux procédés d'évaluation, à l'établissement des valeurs spécifiques dans les diverses substances présentes dans l'environnement et dans les denrées alimentaires, pour lesquelles on peut être amené à introduire des contrôles d'usage ou de consommation. En 1985, l'AIEA a commencé à préparer un document de la "collection sécurité" donnant la ligne de conduite permettant d'établir les niveaux dérivés d'intervention pour les denrées alimentaires et les substances présentes dans l'environnement. A la suite de l'accident de Tchernobyl, ce texte a été révisé pour tenir compte de quelques-unes des leçons et pour disposer d'un document d'orientation plus pratique, contenant des tableaux permettant d'établir des niveaux pour toute une série de denrées alimentaires fraiches ou en conserve. II a été publié en décembre 1986 [7]. 


\section{RÉVISION DES GUIDES INTERNATIONAUX}

9-Au moment de l'accident de Tchernobyl, les guides d'intervention publiés étaient consacrés principalement à l'introduction de mesures de protection dans les phases initiale et intermédiaire d'un accident, la préoccupation principale étant d'éviter les effets non-stochastiques et de limiter l'importance du risque stochastique pour les individus. Cependant, même lors d'un accident nucléaire majeur, impliquant le rejet de quantités importantes de substances radioactives dans l'atmosphère, on circonscrira à des distances relativement faibles du point de rejet (probablement pas plus de quelques dizaines de kilomètres) les mesures de protection destinées à limiter le risque individuel (à savoir mise à l'abri, emploi de médicaments prophylactiques, évacuation). Inversement, parce que les substances radioactives rejetées seront diluées dans l'atmosphère, puis dispersées sur de très vastes étendues, la plus grande partie de la dose collective concernera, en général, des distances beaucoup plus grandes. A ces distances, les niveaux individuels de dose seront très inférieurs à ceux qui entraînent des effets non-stochastiques ou des risques stochastiques individuels significatifs. Néanmoins, les autorités nationales compétentes des pays situés sur la trajectoire des substances radioactives dispersées peuvent encore considérer qu'il est prudent d'essayer de réduire les risques stochastiques pour les individus, ainsi que le détriment collectif pour leurs populations, en prenant des mesures de protection, comme le contrôle des denrées alimentaires et de l'eau de boisson.

10 - Pour éviter que ne puisse se répéter la confusion provenant d'interventions très différentes et résultant, en particulier, de valeurs extrêmement diverses des niveaux pour lesquels ont avait déclenché les mesures de protection, les gouvernements ont demandé à plusieurs organisations internationales ou intergouvernementales:

- de revoir la pertinence des guides existants en ce qui concerne l'application des niveaux de dose déclenchant l'intervention, et d'étudier les critères sur lesquels sont fondées les valeurs numériques des niveaux d'intervention dérivés;

- de compléter ces guides par les valeurs numériques de niveaux d'intervention dérivés (particulièrement pour les denrées alimentaires);

- de rechercher une meilleure harmonisation internationale de l'analyse conduisant à l'établissement des niveaux d'intervention, en particulier pour le commerce international.

Depuis 18 mois, des progrès considérables ont été accomplis dans ces domaines; il existe une coordination étroite entre les organisations intéressées, en particulier l'AIEA, l'Organisation mondiale de la santé (OMS), l'Organisation des Nations Unies pour l'alimentation et l'agriculture (FAO), l'Agence pour l'énergie nucléaire de l'OCDE (AEN), et la CCE. 


\section{L'AIEA}

11 - En février 1987, s'est réuni un groupe consultatif de I'AIEA afin de réviser le guide publié par l'Agence sur les niveaux d'intervention en tenant compte de l'expérience de Tchernobyl. Sa conclusion a été la suivante: bien que les principes de base sur la protection du public donnés dans la "Collection Sécurité n० 72" [4] demeurent valables, ce guide d'orientation avait été surtout conçu dans le contexte d'une intervention au voisinage d'un accident, plutôt que dans le cas d'un accident ayant un impact à longue distance, sur des populations importantes et durant une longue période de temps. Le groupe consultatif en a éclairci et étoffé plusieurs parties; il a précisé les secteurs où il convient de proposer des recommandations supplémentaires. Les principaux domaines abordés sont:

- les critères sur lesquels fonder la mise en œuvre des mesures de protection destinées à diminuer le risque stochastique pour les individus et à limiter le détriment stochastique pour la population exposée (par ex., l'équivalent de dose collectif);

- la nécessité d'exclure des expositions venant d'autres sources que l'accident lui-même, quand on détermine le niveau d'exposition pour lequel on introduit des mesures de protection;

- la nécessité de tenir compte des contraintes spéciales de groupes de la population qui peuvent être particulièrement sujets à risque (par ex., les femmes enceintes, et les personnes qui ont un régime alimentaire exceptionnellement riche en denrées susceptibles d'être contaminées), lorsque l'on détermine le niveau auquel on doit introduire des mesures de protection;

- les mesures qui peuvent s'avérer nécessaires pour contrôler des personnes qui ne sont pas exposées au rayonnement au cours de leur travail mais qui peuvent recevoir une exposition significative, du fait de leurs fonctions particulières en cas d'urgence (par ex., pompiers, ambulanciers, policiers, etc.), ou à cause de la contamination accidentelle de leur environnement de travail;

- les critères fondés sur les principes d'optimisation permettant de réaliser une approche à long terme, harmonisée au niveau international, des problèmes commerciaux en rapport avec d'éventuels accidents nucléaires entraînant une contamination étendue des denrées alimentaires.

12 - Pour déterminer si des mesures de protection seraient justifiées pour réduire l'équivalent de dose collectif à la population, le groupe d'experts a préparé un guide sur l'application des principes d'optimisation de la CIPR en ce qui concerne l'examen du bilan entre le détriment évité par l'introduction d'une mesure de protection, et le coût direct de son introduction. Dans le cas de la contamination des aliments, les calculs préliminaires d'optimisation indiquent que la valeur optimale de la dose ${ }^{1}$ correspondant à l'introduction de mesures de protection se situe probablement entre 1 et $10 \mathrm{mSv}$

1. A strictement parler, la valeur optimale de l'"équivalent de dose efficace engagé". 
pour la nourriture consommée en un an. Elle est relativement indépendante de la séquence de l'accident, du radionucléide concerné, et de l'importance de la population à laquelle s'applique la mesure de protection. Ce niveau est compatible avec les recommandations déjà données dans la "Collection Sécurité $n^{\circ} 72$ " [4], qui recommande un niveau de dose de $5 \mathrm{mSv}$, audessous duquel la mise en œuvre des mesures de protection ne se justifie pas. Le groupe d'experts a noté également que, lorsqu'il s'agit de déterminer les niveaux d'intervention intermédiaires qui s'appliquent au commerce international des denrées alimentaires, un niveau de dose de $5 \mathrm{mSv}$ par an n'est pas incompatible avec ses calculs préliminaires et ses conclusions.

13-II est vivement recommandé que les responsables nationaux chargés de traduire les niveaux d'intervention en limites dérivées à des fins de réglementation prennent garde d'éviter d'intégrer des hypothèses pessimistes pouvant conduire à une dérive importante vis-à-vis des normes de protection contre les rayonnements, à des contradictions majeures dans la pratique de la protection et à des mesures de protection inutilement restrictives et coûteuses. Ainsi, lorsqu'on établit les niveaux d'intervention dérivés à appliquer au commerce international des denrées alimentaires, il faut avoir présent à l'esprit qu'en général, une fraction seulement du panier de la ménagère est importée, et qu'il est probable que, dans cette fraction importée, une quantité limitée provient d'une région contaminée. En général, il ne faudra donc appliquer un niveau d'intervention dérivé qu'à un certain pourcentage de la nourriture totale consommée.

14 - Se fondant sur les recommandations du groupe consultatif, l'AIEA a publié en 1988 un document technique (IAEA-TECDOC) donnant des instructions supplémentaires à l'appui de la "Collection Sécurité $n^{\circ} 72$ " (dont une édition révisée devrait sortir en 1989); elle tiendra compte des commentaires reçus sur le TECDOC, ainsi que des diverses recommandations de la CIPR résultant de la révision actuelle, par un groupe de travail du comité 4 de la CIPR, des recommandations de la publication 40 . Dans ce guide révisé, I'AIEA s'efforce de développer un traitement logique portant sur les trois différentes situations post-accidentelles qui peuvent prédominer, à savoir:

a - la situation au voisinage immédiat du site de l'accident;

b-la situation d'un pays affecté par un dépôt direct de substances radioactives;

$\mathrm{c}$ - la situation où la seule contribution aux doses reçues dans ce pays est due à des denrées alimentaires ou à d'autres produits d'importations.

Pour la première situation (a), on insiste principalement sur la prévention des effets aigus d'un accident (dus à l'exposition au rayonnement externe et à l'inhalation de substances radioactives) et sur la limitation du risque d'effets différés (comme les cancers) pour les individus (par opposition à la population dans son ensemble). Dans la dernière situation (c), on insiste surtout sur la limitation du risque d'effets néfastes pour la population dans son ensemble. Dans la situation (b), intermédiaire entre les situations (a) et (c), il convient de considérer le risque d'effets néfastes pour les individus et pour la population dans son ensemble. 
15 - Parce que les recommandations de la "Collection Sécurité $n^{\circ} 81$ " [7] sur la détermination des niveaux d'intervention dérivés devront tenir compte de ces révisions, cet ouvrage sera également révisé et élargi pour inclure les situations où la voie principale de l'exposition vient davantage des denrées alimentaires importées que d'un dépôt direct. On portera aussi l'attention sur les niveaux d'intervention dérivés pour d'autres voies potentielles de l'exposition, particulièrement celles qui concernent la zone à l'intérieur de laquelle l'accident a un effet immédiat, comme les routes, les étendues de terre, les bâtiments et les vêtements contaminés.

\section{L'OMS}

16 - L'OMS s'intéresse, avant tout, à la santé. Cela se perçoit dans ses activités concernant les niveaux d'intervention dérivés. En avril 1987, un groupe d'expert de l'OMS a ré-éxaminé les divers modes d'établissement des niveaux d'intervention dérivés concernant les denrées alimentaires; il a préparé un projet distribué aux gouvernements pour examen et modification. Après avoir tenu compte des avis reçus, un groupe de travail a revu ce guide; il y a mis un point final en septembre 1987.

17 - En préparant ce guide, on s'est aperçu qu'il ne serait pas possible d'obtenir un consensus universel sur ce qui constituait un détriment "acceptable" pour la santé, ou d'établir des valeurs de niveaux dérivés d'intervention dans les denrées alimentaires uniquement sur la base de l'optimisation des coûts du détriment radiologique (pour la santé) et des mesures de protection imposées. La première décision importante concerne le niveau d'exposition, exprimé en termes de dose, sur lequel doivent se fonder les niveaux dérivés d'intervention. C'est dans la ligne des recommandations faites par la CIPR sur la contamination accidentelle de la nourriture [14] et du guide publié par l'OMS et l'AIEA [22, 4], dans lesquels on avait choisi un engagement d'équivalent de dose de $5 \mathrm{mSv}$, au cours de la première année suivant l'accident, comme niveau au-dessous duquel des mesures de contrôle ne seraient pas justifiées. En pratique, comme la dose individuelle durant les années suivantes sera probablement bien plus faible que celle reçue durant la première année, on ne recommande pas de niveaux de dose plus faibles ni de niveaux d'intervention dérivés pour les années suivantes. Cependant, s'il arrivait qu'une situation particulière n'entraîne pas une diminution significative de la dose dans la seconde ou la troisième année, il appartiendrait aux autorités compétentes de décider toute action nécessaire, fondée sur les circonstances particulières.

18-A un niveau de dose prévisionnel de $5 \mathrm{mSv}$, le risque de cancer mortel sur la durée de la vie (50 ans) est estimé à $10^{-4}$ [13]. Ce degré de risque n'est pas significativement différent de celui associé à l'exposition des populations dans les diverses parties du monde, résultant simplement du bruit de fond naturel et de ses variations. II est aussi comparable au niveau d'exposition à l'intérieur des bâtiments, dû à l'inhalation du radon et de ses. descendants; dans certaines zones, il dépasse $10 \mathrm{mSv}$. 
Bien que le degré de risque calculé pour une exposition de $5 \mathrm{mSv}$ soit de deux ou trois ordres de grandeur supérieur à celui évalué à partir des résultats de la surveillance de routine dans le fonctionnement normal des installations nucléaires, on a considéré qu'un niveau aussi faible n'était pas réalisable en situation accidentelle, quand les autorités doivent faire face à la nécessité d'entreprendre une action rapide, qui, en elle-même, ne doit entraîner ni coût indu, ni détriment pour la santé. Pour la thyroïde, un équivalent de dose efficace de $5 \mathrm{mSv}$ impliquerait un équivalent de dose à cet organe de $167 \mathrm{mSv}$, s'il est le seul organe irradié. On considère que cette dose est trop élevée, étant donné le pouvoir qu'a la thyroïde de concentrer l'iode, et l'incidence d'un cancer non mortel après l'irradiation de la thyroïde. Pour tenir compte de ces facteurs, on a donc recommandé une dose à la thyroïde de $50 \mathrm{mSv}$.

19 - Plutôt que de se fier uniquement aux critères de protection individuelle, on a considéré qu'il fallait aussi estimer le détriment total pour la société afin de juger s'il est possible de le réduire en fixant, pour intervenir, un niveau de dose individuelle plus bas. Le détriment pour la société dépend du nombre des personnes exposées, donc de la dose collective. Si l'on applique le principe de base de la protection radiologique donné par la CIPR, c'est-à-dire maintenir les doses à un niveau "aussi faible que cela est réalisable raisonnablement" (ALARA), il faudra réduire le détriment pour la société jusqu'à un niveau où l'on ne pourra plus justifier aucune autre réduction. On détermine ce niveau en appliquant la technique d'optimisation mise en œuvre par la CIPR: des coûts financiers sont affectés au détriment imputable aux rayonnements et aux mesures de protection. On peut alors calculer le niveau d'intervention pour lequel la somme de ces deux coûts (donc l'impact négatif total sur la société) passe par un minimum. Pourvu que cette valeur calculée soit inférieure au niveau de dose de référence précédemment choisi ( $5 \mathrm{mSv}$ ), on peut l'employer comme nouvelle base de détermination du niveau d'intervention dérivé. Réciproquement, si elle n'est pas inférieure au niveau de la dose de référence, on doit retenir et utiliser ce dernier. En appliquant le procédé de calcul du groupe d'experts de l'AlEA (section 12), on a aussi employé le procédé d'optimisation pour vérifier, d'une manière générale, que le choix de $5 \mathrm{mSv}$ comme niveau de dose de référence constituait une valeur réaliste du point de vue économique.

20 - On peut traduire le niveau de la dose de référence en concentrations correspondantes de radionucléides dans les aliments $(\mathrm{Bq} / \mathrm{kg})$, si l'on connaît les conditions moyennes de consommation d'aliments dans le pays ou la région considérés. On exprime cette information sous la forme de consommation annuelle portant sur les groupes principaux d'aliments, comme les céréales, les légumes, la viande. Les valeurs du guide de l'OMS ont été calculées en compilant les informations globales de la consommation alimentaire; pour établir huit schémas régionaux de consommation alimentaire différents, on s'est appuyé sur des données en provenance d'environ 130 pays. A partir de la consommation régionale maximale pour les différents groupes d'aliments, on a élaboré un régime hypothétique global pour les aliments dont la consommation est supérieure à $20 \mathrm{~kg}$ par personne et par an. On a choisi cette valeur comme limite: pour les aliments 
consommés en plus faibles quantités, il est nécessaire d'avoir des concentrations extrêmement élevées pour atteindre le niveau de dose de référence. Dans le calcul des valeurs des niveaux d'intervention du guide de l'OMS, on a supposé une consommation de $550 \mathrm{~kg}$ d'aliments et de 700 I d'eau de boisson par personne et par an.

21 - II n'est pas possible de prévoir quels radionucléides seront rejetés dans l'environnement quand on établit des schémas généraux; cependant, ceux qui ont le plus de chance d'entrer en jeu sont le strontium 90, l'iode 131 , le césium 134, le césium 137 et le plutonium 239 . Bien que la présence de chacun de ces radionucléides dans les aliments produise une dose différente lorsque les quantités ingérées sont identiques, on divise ces radionucléides en deux groupes. Dans chaque groupe, les différences sont suffisamment faibles pour permettre d'établir une seule série générique de niveaux d'intervention dérivés. Le premier groupe comprend tous les actinides comme le plutonium 239, pour lequel on a prescrit une dose par unité d'activité incorporée de $10^{-6} \mathrm{~Sv} / \mathrm{Bq}$; le second englobe le reste des radionucléides énumérés plus haut comme les césium, pour lesquels on a adopté une valeur générale de $10^{-8} \mathrm{~Sv} / \mathrm{Bq}$ par unité d'activité incorporée. Ces deux facteurs (dose par unité d'activité incorporée) ont été utilisés pour calculer les valeurs guides.

22 - Dans l'éventualité d'un accident, on ne peut prévoir quels seront les aliments contaminés ni quels seront les radionucléides. On a donc fondé les valeurs guides de l'OMS sur l'hypothèse qu'un seul radionucléide est en jeu et qu'un seul groupe d'aliments est contaminé. Le tableau V présente les valeurs guides ainsi calculées. Pour un accident donné, il est probable que plus d'un radionucléide contaminera les aliments de plus d'un groupe alimentaire. Pour prévoir cette éventualité, la méthodologie comprend une formule générale d'additivité, qui établit une répartition des niveaux d'intervention dérivés pour rendre compte de la contamination multiple de l'aliment; elle garantit ainsi que la dose totale ne dépasse pas le niveau de référence de $5 \mathrm{mSv}$. Alors que les valeurs guides doivent protéger correctement l'ensemble de la population, d'autres valeurs supplémentaires sont prévues pour le lait et l'eau consommés par les nourrissons; elles concernent quatre radionucléides et sont fondées sur une consommation de lait et d'eau respectivement de $275 \mathrm{~kg}$ par an.

TABLEAU $V$

Niveaux d'intervention dérivés pour les denrées alimentaires $(\mathbf{B q} / \mathbf{k g})$. Valeurs guides de l'OMS.

\begin{tabular}{|c|c|r|r|r|r|r|r|r|}
\hline $\begin{array}{c}\text { Catégorie de } \\
\text { radionucléide }\end{array}$ & Céréales & Tubercules & Légu. & Fruit & Viande & Lait & Poisson & $\begin{array}{r}\text { Eau de } \\
\text { boisson }\end{array}$ \\
\hline $\begin{array}{c}\text { Facteur de } \\
\text { dose élevé } \\
\left(10^{-6} \text { Sv/Bq) }\right.\end{array}$ & 35 & 50 & 80 & 70 & 100 & 45 & 350 & 7 \\
$\begin{array}{c}\text { Facteur de } \\
\text { dose faible } \\
\left(10^{-8} \text { Sv/Bq) }\right.\end{array}$ & 3500 & 5000 & 8000 & 7000 & 10000 & 4500 & 35000 & 700 \\
\hline
\end{tabular}


Le tableau VI présente ces valeurs, considérées comme nécessaires puisque le régime alimentaire des nourrissons se limite à quelques aliments seulement, et parce que le rapport dose par unité d'activité incorporée pour le strontium 90 et l'iode 131 est plus élevé pour l'enfant.

TABLEAU VI

Niveaux d'intervention dérivés dans le lait et dans l'eau pour les nourrissons $\left({ }^{*}\right)(\mathrm{Bq} / \mathrm{l})$. Valeurs guides de l'OMS.

\begin{tabular}{|l|c|}
\hline \multicolumn{1}{|c|}{ Radionucléide } & Valeur $(\mathrm{Bq} / \mathrm{l})$ \\
\hline Strontium 90 & 160 \\
lode 131 $\left(^{\star \star}\right)$ & $1600\left(^{\star \star \star *}\right)$ \\
Césium 137 & $1800\left(^{\star \star \star *}\right)$ \\
Plutonium 239 & 7 \\
\hline
\end{tabular}

(*) Fondé sur une consommation de lait et d'eau chacune de $275 \mathrm{~kg}$ par an.

(**) Fondé sur une vie moyenne de 11,5 jours et une dose de $50 \mathrm{mSv}$ à la thyroïde.

$(* *)$ Lait uniquement.

23 - Ces recommandations sont conçues pour s'appliquer au point de consommation et à la forme sous laquelle l'aliment est consommé. Par suite de la complexité de la chaîne alimentaire et du fait que les composantes du régime alimentaire de la plupart des individus proviennent de régions différentes, il est probable que seule une fraction de la nourriture consommée se trouve contaminée au niveau qui correspond au niveau de contamination régnant dans la région où ils vivent. En appliquant les critères de contrôle de dose dont nous venons de parler, les doses moyennes obtenues seront plus basses, de manière significative, que le niveau de référence choisi. Dans la pratique, on peut considérer des niveaux spéciaux moins stricts, pour des denrées alimentaires mineures comme les épices, les herbes, le thé, dont il faudrait consommer des quantités importantes avant qu'elles ne contribuent de façon significative au niveau de dose individuelle de $5 \mathrm{mSv}$.

24 - Le guide de l'OMS a pour but d'assister, dans l'exercice de leur jugement, les décideurs en matière de santé publique, qui ne sont pas des spécialistes de la protection contre les rayonnements. L'OMS considère que les valeurs guides seraient particulièrement utiles dans les pays qui ne possèdent pas de programme électronucléaire et donc sans expérience en ce domaine. L'autre objectif a été de fournir une base pour donner à la FAO des avis dans l'établissement de niveaux pour le contrôle de la contamination des denrées alimentaires entrant dans les circuits du commerce international. Le groupe de travail a soumis un rapport à la réunion du conseil exécutif de l'OMS, en janvier 1988; il a été envisagé d'élaborer un projet de résolution et de le soumettre à l'Assemblée mondiale de la santé. L'OMS a prévu de publier ce guide au milieu de 1988; il s'intitule "Les niveaux d'intervention dérivés pour les radionucléides dans les aliments. Guide d'application après une contamination radioactive étendue, résultant d'un accident radiologique majeur" [23]. 


\section{LA FAO}

25 - Le souci général de la FAO est de promouvoir la qualité des aliments, d'assurer la protection du consommateur, et de fournir des avis à ce sujet. Plusieurs États membres de la FAO ont réclamé son avis concernant les actions qu'il serait nécessaire d'entreprendre en ce qui concerne la contamination des aliments par les radionucléides, en particulier pour les denrées impliquées dans le commerce international; en réponse, la FAO a réuni un groupe consultatif d'experts qui a recommandé "les niveaux d'action internationaux provisoires concernant les radionucléides pour les aliments" (IRAFs) [21]. On a employé le terme "provisoire" pour permettre un contrôle périodique et une révision possible, à la lumière d'expériences et de connaissances supplémentaires. Dans l'établissement de ces niveaux, on a adopté une manière de voir relativement prudente (par exemple, pour l'iode 131, $400 \mathrm{~Bq} / \mathrm{kg}$; pour le césium 137, $500 \mathrm{~Bq} / \mathrm{kg}$ la première année, $100 \mathrm{~Bq} / \mathrm{kg}$ les années suivantes); le but est d'avoir des marges de sécurité assez larges, et d'assurer une application internationale aussi grande que possible, pour minimiser des interruptions inutiles du commerce international. On a employé un équivalent de dose efficace engagé de $5 \mathrm{mSv}$ pour la première année d'exposition, et de $1 \mathrm{mSv}$ pour les années suivantes (50 mSv et $10 \mathrm{mSv}$, respectivement, pour des organes spécifiques comme la thyroïde), et l'on a supposé que la nourriture contaminée représentait $100 \%$ de la consommation individuelle. En l'absence d'autres recommandations donnant des niveaux correspondants, la FAO a proposé que les IRAFs soient appliqués aux exportations d'aliments. Elle a considéré aussi que leur application aiderait, entre autres, à protéger la prospérité des communautés agricoles et piscicoles, qui seraient atteintes par de telles interruptions. L'objet des IRAFs de la FAO n'est pas d'exclure l'emploi de niveaux d'intervention dérivés dans les situations d'urgence, ni le développement de tels niveaux par l'OMS ou par d'autres organisations internationales.

26-A la suite de sa distribution à tous les États membres de la FAO, le rapport fut discuté lors de la $20^{e}$ session du comité "Additifs et Contaminants" de la commission "Codex alimentarius", en mars 1987 [1]. Cependant, à cette époque, l'OMS n'avait pas terminé la mise au point des valeurs recommandées pour les niveaux d'intervention dérivés; le rapport a donc été soumis "pour information" à la $17^{\mathrm{e}}$ session de la commission "Codex alimentarius" en juin 1987. De la réunion inter-secrétariat de la FAO et de l'OMS en mars 1988, il est résulté une proposition commune concernant les niveaux de contamination radioactive des aliments faisant l'objet d'échanges internationaux, elle a été soumise au conseil de la commission "Codex alimentarius" en juillet 1988. Ces propositions seront, nous l'espérons, largement distribuées aux États membres de la FAO et de l'OMS avant le congrès de mars 1989 du comité "Additifs et Contaminants" du "Codex alimentarius" qui étudiera ces propositions et les commentaires qu'il aura reçus ${ }^{1}$. Le tableau VII présente les niveaux proposés (et les valeurs initiales IRAF); il les compare aux niveaux d'intervention dérivés adoptés par l'OMS et la CCE.

1. Les auteurs donnent la situation en avril 1988 (NdT). 
NIVEAUX D'INTERVENTION POUR LA PROTECTION DE LA POPULATION






\section{L'AEN}

27 - A la suite de l'accident de Tchernobyl, l'AEN a effectué, de manière indépendante, l'évaluation de ses conséquences radiologiques et mené en même temps une étude critique des ripostes face à une situation d'urgence dans les pays membres de l'AEN. Les résultats figurent dans le rapport: "Impact radiologique de l'accident de Tchernobyl dans les pays de l'OCDE" préparé sous l'égide du comité "Radiation protection and public health" (CRPPH), sur la base des informations fournies par les pays de l'OCDE membres de l'AEN [6]. Cette étude représente une première étape vers l'identification des secteurs que l'on doit considérer pour tirer des enseignements des réactions et de l'expérience résultant de l'accident de Tchernobyl.

28 - L'AEN a également mis en place un groupe d'experts CRPPH sur les niveaux d'intervention face à des situations d'urgence nucléaire; sa mission est de revoir les ripostes et les niveaux d'interventions primaire et dérivé correspondants, adoptés par les pays membres, d'examiner la possibilité d'établir des critères d'intervention dérivés, enfin de fournir des instructions qui permettent d'améliorer l'harmonisation internationale de ces niveaux d'intervention. Le groupe a entrepris d'identifier les paramètres clefs, influant sur le processus de la prise de décision et impliqués dans la maîtrise de la situation d'accident. II a considéré aussi la possibilité d'une meilleure harmonisation des critères de protection qui gouvernent les décisions sur les mesures de protection, y compris la possibilité d'harmoniser les valeurs numériques. II a aussi considéré la possibilité d'appliquer le processus d'optimisation au développement de niveaux d'intervention; il a apporté sa contribution au groupe de travail de l'OMS chargé de rédiger un guide sur les niveaux d'intervention dérivés. On espère que le rapport de l'AEN sera publié en 1988 [8].

\section{CCE}

29 - A la suite de l'accident de Tchernobyl, la Commission a recommandé, au début de mai 1986, des niveaux de protection pour l'iode 131. A la fin de mai, le Conseil des ministres de la CCE a donné son accord sur les niveaux de concentration maximale pour le césium 134 et le césium 137 dans les produits agricoles importés de pays extérieurs à la Communauté européenne. II a aussi donné son accord pour que les niveaux concernant le commerce intérieur à la Communauté ne soient pas inférieurs à ceux recommandés pour les importations [10]. L'article 31 du traité d'Euratom prévoit qu'un groupe d'experts conseille la Commission sur la protection contre les rayonnements. En 1987, utilisant "un panier de la ménagère" simplifié et regroupant des isotopes, ce groupe d'experts a recommandé un système de niveaux facile à appliquer, pouvant être employé lors de tout accident futur. Cela servirait de mesure provisoire, jusqu'à ce que l'on ait procéder à un examen détaillé de la situation, afin de déterminer la nécessité de contrôles plus adaptés à ces circonstances particulières. 
Se fondant sur les recommandations du groupe d'experts, la Commission a formulé une proposition pour l'établissement d'une réglementation à adopter par le Conseil des ministres, établissant un système à deux échelons de niveaux maximaux admissibles de contamination radioactive autorisés, pour les denrées alimentaires, l'eau de boisson, les aliments pour bétail, qui puisse s'appliquer rapidement dans le cas de niveaux anormaux de radioactivité ou d'accident nucléaire [11].

30 - Sur proposition de la Commission, le Conseil a décidé que les valeurs acceptées antérieurement (par ex., dans le cas du césium 134 et du césium 137, $370 \mathrm{~Bq} / \mathrm{kg}$ pour le lait et les aliments pour nourrissons, $600 \mathrm{~Bq} / \mathrm{kg}$ pour les autres denrées alimentaires) devaient rester en vigueur durant une période supplémentaire de deux ans. II a aussi adopté une réglementation établissant les principes d'un système permanent permettant l'introduction automatique de niveaux maximaux autorisés de contaminants radioactifs dans les denrées alimentaires et les aliments pour bétail qui seraient placés sur le marché après un accident nucléaire, ou dans toute situation d'urgence radiologique entraînant la contamination de la nourriture. Ces niveaux sont fondés sur les avis du groupe d'experts (tableau VII). Le système d'urgence déclenché, l'application des niveaux ne devrait pas dépasser une période maximale de trois mois; durant cette période, la Commission, après avis du groupe d'experts, établit les limites spécifiques pour les circonstances particulières de l'accident [12].

\section{HARMONISATION FUTURE}

31 - La confusion et l'anxiété du public après l'accident de Tchernobyl ont été, en grande partie, le résultat d'un mode d'approche incohérent entre les pays, et dans certains cas à l'intérieur des pays, en ce qui concerne l'interprétation et l'application des critères radiologiques sur lesquels fonder la mise en œuvre des mesures de protection. Sans aucun doute, y ont contribué le manque de recommandations publiées sur l'application des niveaux d'intervention et l'établissement de niveaux d'intervention dérivés, particulièrement pour des situations où la préoccupation majeure concerne la limitation de la dose collective. Les progrès effectués depuis dix-huit mois indiquent que l'on a beaucoup fait pour combler ces omissions; il existe maintenant une base commune solide sous-tendant les recommandations et les guides élaborés par les organisations internationales et inter-gouvernementales. Non seulement il existe un large consensus sur les principes à appliquer pour établir les niveaux d'intervention, mais il y a aussi un bon accord entre les facteurs - mais non pas tous - à appliquer quand on détermine les niveaux dérivés d'intervention, particulièrement lors de leur application dans les processus d'optimisation.

32 - II est hors de doute que les domaines les plus importants où les directives font encore défaut sont liés au commerce et à la consommation de denrées alimentaires contaminées. Cependant, quand on considère la possibilité d'une harmonisation future, il est essentiel de faire la différence entre les niveaux à établir en vue de mesures de protection destinées à 
éviter ou à minimiser les conséquences sanitaires (pour les individus ou pour des groupes de population), et ceux qui sont nécessaires pour rendre aussi faible que possible toute entrave au commerce international. Les deux doivent avoir en commun les mêmes principes de base d'intervention; cependant, il faut que tout régime de contrôle concernant le commerce international soit facile à comprendre et à mettre en œuvre par des nonspécialistes en radioprotection. Les plus adaptés à cet objectif sont les protocoles (comme ceux mis au point conjointement par la FAO et l'OMS) qui donnent un seul niveau (plutôt qu'une série) à partir duquel il faut agir; ce niveau est fondé sur un nombre minimal de radionucléides et de groupes de denrées alimentaires. Le handicap de toute approche simplifiée pour fixer des niveaux d'action est qu'il peut s'avérer nécessaire d'assigner à certains paramètres de base des valeurs plus prudentes qu'il n'est justifié du strict point de vue de la radioprotection. Même s'il en est ainsi, le surcoût de la protection qui en résulte peut être considéré comme un sacrifice "acceptable" quand on le compare au bénéfice qu'offre un contrôle harmonieux des denrées alimentaires contaminées qui transitent dans le commerce international.

33 - Par contre, on peut ne pas réaliser aussi facilement l'harmonisation des niveaux d'intervention dérivés sur lesquels fonder la mise en œuvre de mesures pour la protection du public, en particulier en ce qui concerne la consommation des denrées alimentaires. Bien que le guide et les niveaux qui vont être publiés par l'OMS (tableaux $V$ et $\mathrm{VI}$ ) aient été établis en liaison avec d'autres organisations internationales et inter-gouvernementales, tous les pays et les organisations inter-gouvernementales ne sont pas préparés à adopter les niveaux recommandés s'ils diffèrent de manière significative de ceux qu'ils emploient déjà, particulièrement s'ils se trouvent déjà formulés dans une législation nationale. Même là où l'on peut réaliser un large degré d'harmonisation, on doit toujours introduire une souplesse suffisante pour tenir compte des circonstances spécifiques de l'accident, et de la zone où il est nécessaire d'introduire les mesures de protection. Depuis l'accident de Windscale, l'élaboration des principes de protection du public dans l'éventualité d'un accident nucléaire et la détermination des niveaux auxquels il faut déclencher cette protection a beaucoup avancé. Nous ne sommes pas encore parvenus à un système d'intervention totalement harmonisé, mais les progrès déjà réalisés pour aboutir à un accord international montrent que nous sommes dans la bonne voie.

\section{RÉFÉRENCES}

[1] AGENCE INTERNATIONALE DE L'ÉNERGIE ATOMIQUE (AIEA). - Annual nuclear safety review, 1987, Vienne: AIEA, 1988.

[2] AGENCE INTERNATIONALE DE L'ÉNERGIE ATOMIQUE (AIEA). - Normes fondamentales de radioprotection (Collection sécurité $n^{\circ}$ 9). Vienne: AIEA, 1982.

[3] AGENCE INTERNATIONALE DE L'ÉNERGIE ATOMIQUE (AIEA). - Planning for off-site response to radiation accidents in nuclear facilities (Safety Series $n^{\circ} 55$ ). Vienne: AIEA, 1981. 
[4] AGENCE INTERNATIONALE DE L'ÉNERGIE ATOMIQUE (AIEA). - Principles for establishing intervention levels for the protection of the public in the event of a nuclear accident or radiological emergency (Safety Series $n^{\circ} 72$ ), AIEA, 1985.

[5] AGENCE INTERNATIONALE DE L'ÉNERGIE ATOMIQUE (AIEA). - Summary report on the post-accident review meeting on the Chernobyl accident (Safety Series $n^{\circ}$ 75-INSAG-1), Vienne: AIEA, 1986.

[6] AGENCE POUR L'ÉNERGIE NUCLÉAIRE DE L'OCDE (AEN). - The radiological impact of the Chernobyl accident in OECD countries. Paris: OCDE, 1987.

[7] AGENCE INTERNATIONALE DE L'ÉNERGIE ATOMIQUE (AIEA). - Derived intervention levels for application in controlling radiation doses to the public in the event of a nuclear accident or radiological emergency - principles, procedures and data (Safety Series $n^{\circ} 81$ ). Vienne: AIEA, 1986.

[8] AGENCE POUR L'ÉNERGIE NUCLÉAIRE DE L'OCDE (AEN). - Critical review of intervention levels for protection of the public following nuclear accidents. Paris: OCDE (à paraître).

[9] COMMISSION DES COMMUNAUTÉS EUROPÉENNES (CCE). - Critères de radioprotection pour limiter l'exposition du public en cas de rejet accidentel de substances radioactives. Guide de niveaux de référence établi par le groupe d'experts institué par l'article 31 du traité d'Euratom. Luxembourg: CCE, 1982.

[10] COMMISSION DES COMMUNAUTÉS EUROPÉENNES (CCE). - Recommandation de la Commission du 6 mai 1986, décision du 7 mai 1986 et règlements des 12, 16 et 30 mai 1986, concernant les mesures nationales prises à l'égard des produits agricoles. J. Off. Comm. Europ., mai 1986.

[11] COMMISSION DES COMMUNAUTÉS EUROPÉENNES (CCE). - Proposition de règlement (Euratom) du Conseil fixant les niveaux maximaux admissibles de radioactivité pour les denrées alimentaires, les aliments pour bétail et l'eau potable en cas de niveaux anormaux de radioactivité ou d'accident nucléaire (COM (87) 281 final; 87/C 174/09). J. Off. Comm. Europ., 1987, C 174, 6-8.

[12] COMMISSION DES COMMUNAUTÉS EUROPÉENNES (CCE): Résolution du Conseil adoptée à l'occasion de l'adoption du règlement (EURATOM) N. 3954/87 du Conseil, du 22 décembre 1987, fixant les niveaux maximaux admissibles de contamination radioactive pour les denrées alimentaires et les aliments pour bétail après un accident nucléaire ou dans toute autre situation d'urgence radiologique). J. Off. Comm. Europ., 1987, C 352, 1.

[13] COMMISSION INTERNATIONALE DE PROTECTION RADIOLOGIQUE (CIPR). Recommandations de la Commission internationale de protection radiologique (Publication CIPR $n^{\circ} 26$ ). Oxford: Pergamon press, 1977.

[14] COMMISSION INTERNATIONALE DE PROTECTION RADIOLOGIQUE (CIPR). Protection of the public in the event of major radiation accidents: principles for planning (ICRP Publication $n^{\circ}$ 40). Oxford: Pergamon Press, 1984.

[15] COUNCIL FOR MUTUAL ECONOMIC ASSISTANCE (CMEA). - Criteria for radioactive releases from nuclear power plants requiring other countries to be informed (Radiation Protection Series $n^{\circ}$ 18). Moscou: CMEA, 1984. (en russe).

[16] GRANDE BRETAGNE. ATOMIC ENERGY OFFICE. - The accident at the Windscale No 1 pile on 10 October 1957 (Command 302). London: HMSO, 1957.

[17] GRANDE BRETAGNE. MEDICAL RESEARCH COUNCIL. - Criteria for controlling radiation doses to the public after accidental escape of radioactive material. London: HMSO, 1975. 
[18] GRANDE-BRETAGNE. NATIONAL RADIOLOGICAL PROTECTION BOARD (NRPB). - Derived emergency reference levels for the introduction of countermeasures in the early to intermediate phases of emergencies involving the release of radioactive materials to atmosphere (NRPB-DL 10). London: HMSO, 1986.

[19] ILYIN L. A., PAVLOVSKIJ O.-A. - Conséquences radiologiques de l'accident de Tchernobyl en Union Soviétique et mesures prises pour en atténuer l'impact. AIEA Bull. 1987, 29 (4), 17-24.

[20] KEMENY J.-G. - The President's commission on the accident at Three Mile Island. New York: Pergamon Press, 1979.

[21] ORGANISATION DES NATIONS-UNIES POUR L'ALIMENTATION ET L'AGRICULTURE (FAO). - Recommended limits for radionuclide contamination of foods, report of an expert consultation, Rome, décembre 1986.

[22] ORGANISATION MONDIALE DE LA SANTÉ (OMS), BUREAU RÉGIONAL POUR L'EUROPE. - Energie nucléaire: rejets accidentels - principes applicables aux mesures de santé publique. Copenhague: OMS, Bureau régional pour l'Europe, 1984.

[23] ORGANISATION MONDIALE DE LA SANTÉ (OMS). - Derived intervention levels for radionuclides in food, guidelines for application after widespread radioactive contamination resulting from a major radiation accident, Genève: OMS (1988).

[24] SALO A. - Radiation levels in Europe after Chernobyl, meeting of the European thyroid association, July 1987.

[25] WHITE I.F., NRPB. - Emergency data handbook. NRPB - R182, 1986. 